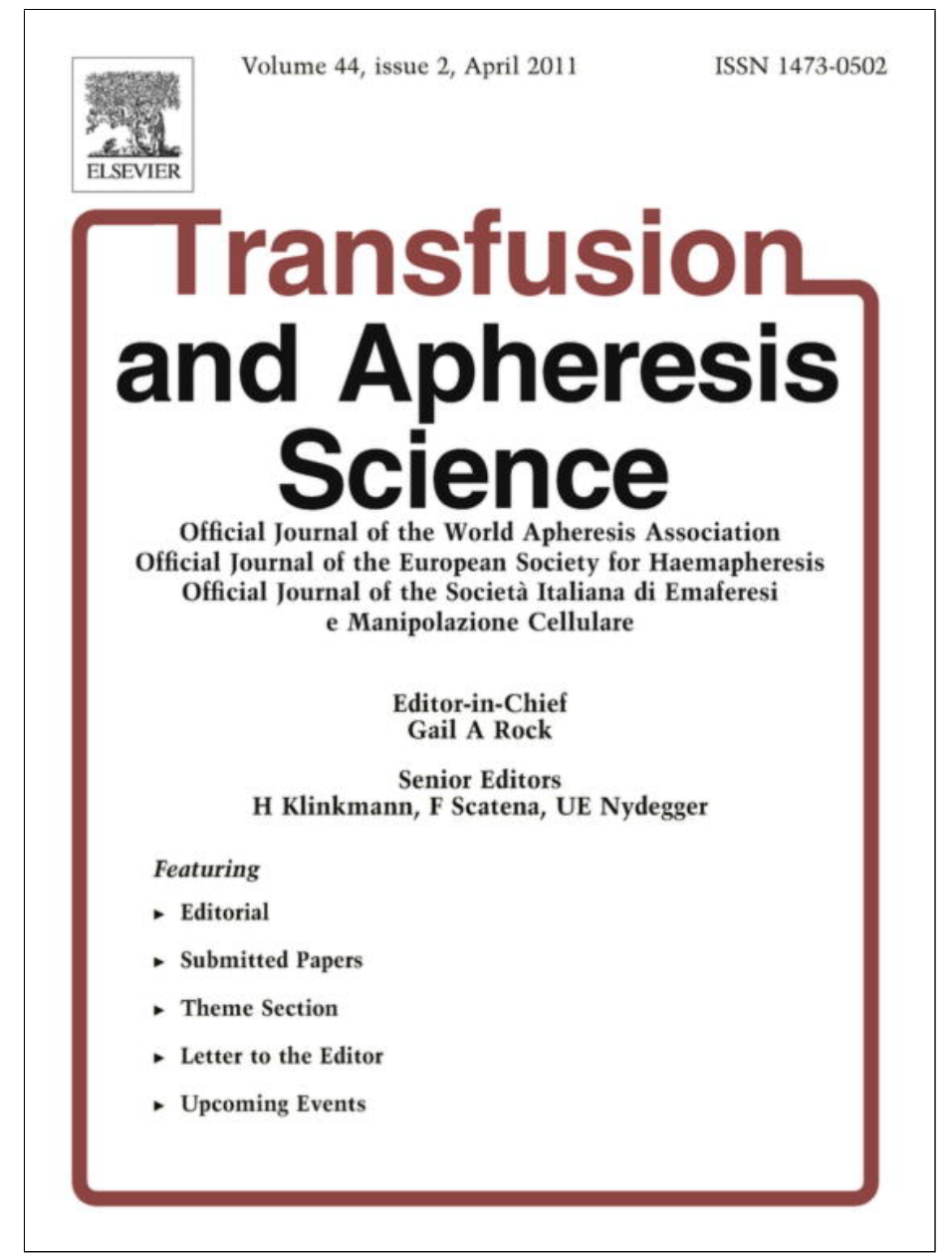

This article appeared in a journal published by Elsevier. The attached copy is furnished to the author for internal non-commercial research and education use, including for instruction at the authors institution and sharing with colleagues.

Other uses, including reproduction and distribution, or selling or licensing copies, or posting to personal, institutional or third party websites are prohibited.

In most cases authors are permitted to post their version of the article (e.g. in Word or Tex form) to their personal website or institutional repository. Authors requiring further information regarding Elsevier's archiving and manuscript policies are encouraged to visit:

http://www.elsevier.com/copyright 


\title{
Allogeneic hematopoietic stem cell transplantation (HSCT) after reduced intensity conditioning
}

\author{
Sophie Servais, Frédéric Baron, Yves Beguin* \\ Department of Medicine, Division of Hematology, University of Liège, Liège, Belgium \\ Giga-Research, Section of Hematology, University of Liège, Liège, Belgium
}

\section{A R T I C L E I N F O}

\section{Keywords:}

Reduced-intensity conditioning

Hematopoietic stem cell transplantation

Graft-versus-tumor effects

Graft-versus-host disease

\begin{abstract}
A B S T R A C T
Allogeneic hematopoietic stem cell transplantation (HSCT) following myeloablative (conventional) conditioning regimen is associated with a high incidence of transplant-related morbidity and mortality, limiting its use to younger patients without medical co-morbidities. Over the past few years, it has become more evident that the alloreactivity of transplanted donor immunocompetent cells against host tumor cells (graft-versus-tumor effects, GVT effects) plays a major role in eradicating malignancies after allogeneic HSCT. Based on these observations, several groups of investigators have developed reduced intensity conditioning (RIC) regimens allowing patients who are ineligible for conventional HSCT to benefit from the potentially curative GVT effects of allogeneic transplantation. Retrospective studies have suggested that, in comparison with myeloablative allogeneic HSCT, in patients aged 40-60 years, RIC HSCT was associated with a higher risk of relapse but a lower incidence of transplant-related mortality leading to similar progression-free and overall survivals. Prospective studies are ongoing to define which patients might most benefit from RIC HSCT, and to increase the anti-tumoral activity of the procedure while reducing the incidence and the severity of acute graft-versus-host disease (GVHD). In this article, we review the current status and perspectives of RIC HSCT.
\end{abstract}

(C) 2011 Elsevier Ltd. All rights reserved.

\section{Introduction}

Allogeneic hematopoietic stem cell transplantation (HSCT) following myeloablative conditioning is an effective therapy for many patients with hematological diseases. However, myeloablative conditioning regimens have been associated with significant morbidity and mortality, particularly in patients older than 45 years, those affected by other comorbidities or who have been heavily pretreated (more than two lines of conventional chemotherapy or previous HSCT). This is why conventional allogeneic HSCT has been restricted to younger patients $(<50-55$ years of age) in good medical condition, whereas the median

\footnotetext{
* Corresponding author. Address: Department of Hematology, University of Liège, CHU Sart-Tilman, 4000 Liège, Belgium. Tel.: +32 436672 01; fax: +324366 8855 .

E-mail address: Yves.Beguin@chu.ulg.ac.be (Y. Beguin).
}

patient age at diagnosis for many hematological malignancies is over 50 years [1].

Allogeneic HSCT initially relied on maximally tolerated doses of systemic chemo-radiotherapy to eradicate malignant cells, while hematopoietic stem cells (HSC) were infused to rescue patients from lethal myeloablation that occured as side effect [1]. However, it was quickly recognized that the allograft itself conferred immune-mediated antileukemic effects, termed graft-versus-tumor (GVT) effects [2]. The recognition of the importance of GVT effects in the eradication of malignant cells after myeloablative allogeneic HSCT led to the development of reduced intensity (RIC) or truly nonmyeloablative conditioning regimens.

RIC allogeneic HSCT is associated with reduced regimen-related toxicities and reduced transplant-related mortality rates. Therefore, it allows patients who are ineligible for conventional allogeneic HSCT to benefit from the potentially curative GVT effects of allogeneic HSCT. 
In this article, we would like to propose a brief review of this type of allografting by focusing on the GVT effect, RIC regimens commonly used, the kinetics of engraftment, the incidence and impact of acute and chronic GVHD, and the current results of RIC HSCT in specific diseases.

\section{Definition and features of RIC allogeneic HSCT}

\subsection{Graft-versus-tumor effect}

The concept of GVT effects after allogeneic HSCT is supported by several lines of clinical evidence: (1) patients who develop graft-versus-host disease (GVHD) have a lower risk of relapse than those who do not, (2) patients given T-cell depleted grafts or those given grafts from identical twins have higher risks of relapse, and (3) donor lymphocyte infusions can induce complete remissions (CR) in a number of patients who have relapsed hematological malignancies after allogeneic HSCT [3,4].

It has been suggested that the main mechanisms of GVT effects were the recognition of host-specific minor or major histocompatibility antigens (and maybe tumorspecific antigens) by donor T cells, although several observations have also suggested a role for donor NK and B cells [5-9].

After RIC allogeneic HSCT, tumor eradication relies partially (RIC regimens) or nearly entirely (truly nonmyeloablative regimens) on these GVT effects. Antitumor responses may require extended periods of time, with some patients achieving complete remission more than 1 year after HSCT. GVT activity may also vary in intensity depending on the immunogenicity of the tumors and the respective proliferation rates of tumor cells and donor immune cells [10].

\subsection{Nonmyeloablative versus reduced intensity conditioning regimens}

In comparison with conventional myeloablative conditioning, RIC regimens have been developed with the aim to induce only reversible myelosuppression and less nonhematological toxicity. These regimens exert only low or moderate activity against malignant cells but provide sufficient immune suppression to prevent immediate rejection of the graft and to allow GVT effects [11].

All RIC regimens should not be considered equivalent, since their immunosuppressive and myelosuppressive properties may be different. In particular, one can distinguish RIC regimens and truly nonmyeloablative regimens.

Most RIC regimens combine fludarabine with intermediate doses of alkylating agents such as busulfan, melphalan or thiothepa. These regimens limit graft rejection (fludarabine) but also convey major antitumor effects by themselves with the aim of shrinking and controlling the malignancy (alkylating agents) before the GVT effects can occur [12]. Typical complications of high-dose therapy, such as mucositis, pancytopenia and organ damage can be observed with these RIC regimens but occur less frequently than after myeloablative conditioning.
In contrast, nonmyeloablative regimens rely on optimization of pre- and post-transplant immunosuppression to allow engraftment, while eradication of tumors depends nearly exclusively on GVT effects. The most common nonmyeloablative regimen consist of low-dose (2 Gy) total body irradiation (TBI) with or without fludarabine combined with postgrafting immunosuppression with mycophenolate mofetil (MMF) and cyclosporine (CSP). Other regimens associate cyclophosphamide and fludarabine, or anti-thymocyte globulin (ATG) and total lymphoid irradiation (TLI) (8 Gy) with postgrafting immunosuppression also consisting in MMF and CSP [12]. These types of conditioning regimens produce only mild myelosuppression and little regimen-related toxicity, making them realizable in an outpatient setting [13]. Following nonmyeloablative conditioning, antitumor responses may require extended periods of time. Because of that, nonmyeloablative HSCT is generally not recommended for patients with aggressive malignancies not in good response at the time of HSCT.

It has been suspected that RIC regimens might be associated with better survival than truly nonmyeloablative conditioning because of a possibly lower incidence of relapse and similar non-relapse mortality. However, two recent studies have suggested that it might not be the case. Blaise et al. reported the results of a prospective randomized study comparing outcomes of 139 patients with hematological malignancies given grafts after fludarabine, busulfan $(8 \mathrm{mg} / \mathrm{kg})$ and ATG $(n=69)$ or fludarabine and 2 Gy TBI $(n=70)$ [14]. With a median follow-up of 4 years, overall survival (the primary endpoint of the study) was similar in the two arms (44\% versus $47 \%$, respectively). Further, Mohty et al. compared outcomes of patients with acute myeloid leukemia (AML) in first CR given grafts following low-dose TBI-based nonmyeloablative conditioning $(n=323)$ versus more intense but still RIC regimens $(n=877)$ in European group for Blood and Marrow Transplantation (EBMT)-affiliated centers [15]. Two-year disease-free survival was similar in the two groups $(50 \%$ versus 53\%, respectively).

\subsection{Engraftment: mixed chimerism and risk of graft rejection}

In contrast with allogeneic transplantation after myeloablative conditioning, RIC HSCT usually leads to an initial state of mixed chimerism defined as the coexistence in the bone marrow of hematopoietic cells from host and from donor origin. Eventually, the majority of patients evolve to full donor chimerism. Achievement of full donor T-cell chimerism is strongly correlated with a decreased risk of progression or relapse [16]. Therefore, attempts to convert mixed chimerism into full donor chimerism (for example by using donor lymphocyte infusions) should be considered in some cases.

RIC HSCT is associated with a higher risk of graft rejection compared with conventional regimens. McSweeney et al. reported a $20 \%$ graft rejection rate $2-4$ months after HSCT with TBI alone as nonmyeloablative conditioning regimen [17]. High levels ( $>50 \%$ ) of donor T- and NK-cell chimerism one month after transplantation are associated with a lower risk of graft rejection [13]. 


\section{Transplantation-related morbidity}

\subsection{Graft-versus-host disease (GVHD)}

A number of reports have compared the incidences of acute and chronic GVHD after RIC and after myeloablative allogeneic HSCT. Most have shown a lower incidence of acute GVHD (when acute GVHD was defined as GVHD occurring before day 100) but a similar incidence of chronic GVHD with RIC regimens [18]. The relatively reduced incidence and severity of acute GVHD after RIC transplantation can be explained by the combination of low-intensity pre-transplant conditioning and the initial mixed chimerism that may favor both host-versus-graft and graft-versus-host tolerance $[17,18]$. However, a number of patients given RIC conditioning experience late acute GVHD (i.e. acute GVHD occurring after day 100), often at the time of conversion from mixed to full donor Tcell chimerism $[13,18]$.

Occurrence of GVHD is strongly associated with graftversus-tumor effects in patients given myeloablative conditioning [2]. Since nonmyeloablative regimens rely nearly exclusively on GVT effects for tumor eradication, several groups of investigators looked at the impact of GVHD on HSCT outcomes after nonmyeloablative or RIC conditioning. The Seattle group analyzed the impact of acute and chronic GVHD on HSCT outcomes in a cohort of 322 patients given nonmyeloablative HSCT as treatment for various hematological malignancies [16]. Grade II and grade III-IV acute GVHD were not significantly associated with lower risks of progression/relapse, but were instead associated with increased non-relapse mortality and lower progression-free survival. In contrast, occurrence of chronic GVHD correlated with lower risks of relapse in multivariate time-dependent analyses $(\mathrm{HR}=0.4, P=0.006)$ and was associated with significantly better progression-free survival ( $\mathrm{HR}=0.5, P=0.003$ ). Similar observations were reported by Valcarcel et al., in a cohort of 93 patients given grafts after RIC as treatment for AML or advanced myelodysplastic syndrome (MDS) [19].

\subsection{Other toxicities}

The profound pancytopenia following myeloablative conditioning is less profound after RIC regimens and patients require fewer platelet and red blood cell transfusions [17,20].

In a high proportion of patients, neutrophil counts remain over $0.5 \times 10^{9}$ cells/L during the transplant period or the duration of neutropenia is very short (median time 13 days) [20,21]. This is associated with a lower risk of early bacteremia $[21,22]$. There is no difference in the incidence of fungal infections after RIC transplantation compared with myeloablative conditioning and CMV manifestations are just delayed [21,22].

RIC regimens are well tolerated in terms of mucosal toxicity and tissue (liver, kidney, gastrointestinal tract and lung) damages are significantly reduced $(1,23)$. Most of them can also prevent some classical long-term toxicity of conventional conditioning regimens (e.g. preservation of fertility).

\section{Outcomes and anti-tumoral activity}

\subsection{Comparison of outcomes after myeloablative or RIC regimens}

Three large retrospective studies from the EBMT have compared HSCT outcomes of patients given various myeloablative versus various RIC/nonmyeloablative regimens as treatment for AML, MDS, or chronic lymphocytic leukemia (CLL) (Table 1) [23-25]. Obviously these studies are limited by the fact that fitter patients were probably more often proposed myeloablative regimens, while older and sicker patients were given nonmyeloablative or RIC regimens. Nevertheless, these studies found similar disease-free and overall survivals in the two groups of patients, since non-relapse mortality was lower in nonmyeloablative patients, but relapse rates were lower in myeloablative recipients [23-25].

\subsection{Results in specific diseases}

\subsubsection{Acute leukemias}

The largest prospective study of nonmyeloablative HSCT as treatment for AML has been recently reported by the Seattle consortium for 274 patients with AML in first $(n=160)$ or second $(n=71)$ CR or with more advanced diseases $(n=43)$ [26]. The conditioning regimen consisted in fludarabine and $2 \mathrm{~Gy}$ TBI. Five-year overall survival was $37 \%$ for patients in first CR at the time of HSCT, 34\% for those in second CR and 18\% for those with more advanced disease. Other factors associated with survival included cytogenetic risk (5-year overall survival of $40 \%$ for patients with good/intermediate cytogenetics versus $19 \%$ for those with poor risk cytogenetics), and chronic GVHD that was associated with better survival in time-dependent analysis ( $\mathrm{HR}=0.7, P=0.07$, due to a $50 \%$ reduction in the risk of re-

Table 1

Retrospective studies comparing HSCT outcomes of patients given grafts after nonmyeloablative/RIC or myeloablative conditioning

\begin{tabular}{lllll}
\hline $\begin{array}{l}\text { Authors } \\
\text { (reference) }\end{array}$ & Disease & $\begin{array}{l}\text { Relapse (HR (95\%CI); } P \\
\text { value) }\end{array}$ & $\begin{array}{l}\text { Non-relapse mortality (HR (95\%CI), } \begin{array}{l}\text { Progression-free survival (HR (95\%CI), } P \\
\text { value) }\end{array} \\
\text { value) }\end{array}$ \\
\hline $\begin{array}{llll}\text { Aoudjhane [23] } \\
\text { Martino [25] }\end{array}$ & AML & $1.78(1.30-2.43) ; 0.0003$ & $0.48(0.33-0.68) ;<0.001$ & $1.15(0.90-1.47) ; 0.24$ \\
Dreger [24] & CLL & $2.46(0.90-6.72) ; 0.08$ & $0.40(0.18-0.90) ; 0.03$ & $1.10(0.80-1.40) ; 0.9$ \\
$0.69(0.38-1.25) ; 0.22$
\end{tabular}

AML, acute myeloid leukemia; MDS, myelodysplastic syndrome; CLL, chronic lymphocytic leukemia; HR, hazard ratio. 
lapse $(P=0.01))$. The later observations clearly demonstrate that AML is highly susceptible to GVT effects.

Nonmyeloablative or RIC HSCT is also increasingly used as treatment for patients with acute lymphoblastic leukemia (ALL). Interestingly, preliminary data from two retrospective studies (one from the CIBMTR and one from the EBMT) have showed similar survival in ALL patients given RIC/nonmyeloablative conditioning compared with those given myeloablative conditioning [27]. These encouraging results confirm the existence of graft-versus-ALL effects.

\subsubsection{Myelodysplastic syndromes and myeloproliferative disorders}

Martino et al. reported the outcomes of 215 patients given one of various RIC or nonmyeloablative conditioning regimens as treatment for MDS (refractory anemia $(n=20)$ or more advanced MDS $(n=195))$ in EBMT-affiliated centers [25]. Three-year incidences of relapse and non-relapse mortality were $45 \%$ and $22 \%$, respectively, while 3 -year overall survival was $41 \%$. These results seem to be better than those reported by Laport et al. in patients with MDS given grafts after $2 \mathrm{~Gy}$ TBI alone or with fludarabine (3-year relapse rate of $41 \%$ and 3 -year overall survival of $27 \%$ ), perhaps due to a high incidence of graft rejection among previously untreated patients given lowintensity nonmyeloablative conditioning [28].

More recently, Kroger et al. reported the results of a prospective study of RIC allogeneic HSCT as treatment for 103 patients with myelofibrosis [29]. The conditioning regimen consisted in busulfan $(10 \mathrm{mg} / \mathrm{kg})$, fludarabine and ATG. The 5-year incidence of relapse was $29 \%$, while 5 -year progression-free and overall survivals were $51 \%$ and $67 \%$, respectively.

\subsubsection{Lymphomas}

A number of studies have evaluated HSCT following reduced intensity or nonmyeloablative conditioning regimens as treatment for patients with advanced lymphoma, including those relapsing after autologous HSCT [30-33]. Graft-versus-tumor effects were particularly impressive in patients with indolent or mantle cell lymphoma, as well as in those with chemosensitive aggressive lymphomas [10]. Further, two recent reports demonstrated that some patients with Hodgkin's lymphoma relapsing after autologous HSCT could be salvaged with nonmyeloablative/RIC allo-HSCT [34].

\subsubsection{Chronic lymphocytic leukemia}

Sorror et al. described outcomes in 152 patients (median age 60 years) with CLL (40) or lymphoma (112) who received HSCT from HLA-matched related $(n=84)$ or unrelated $(n=68)$ donors after conditioning with $2 \mathrm{~Gy}$ TBI alone or combined with fludarabine [35]. With a median follow-up of 4 years, the 3-year rate of non-relapse mortality was $25 \%$, while 3 -year overall survival was $53 \%$. These results demonstrate that CLL is remarkably susceptible to GVT effects, and that a significant proportion of patients with fludarabine-refractory CLL might be cured by RIC/nonmyeloablative HSCT.

\subsubsection{Multiple myeloma}

The role of nonmyeloablative HSCT in patients with multiple myeloma has been controversial. The Intergroupe Francophone du Myelome compared the outcomes of 284 patients with high risk multiple myeloma treated in two separate prospective protocols consisting of either double autologous HSCT or single autologous HSCT followed by RIC allogeneic HSCT [36]. Survival was worse in the autologous/allogeneic arm but this might be due to the fact that allografting was preceded by administration of high doses of ATG that could have abrogated graft-versus-myeloma effects. Bruno et al. compared a protocol that entailed an autologous HSCT followed by an allograft from an HLAidentical sibling (following conditioning with $2 \mathrm{~Gy} \mathrm{TBI}$ ) for all patients with a HLA-identical sibling with a protocol of tandem autologous HSCT for patients without a HLAidentical sibling [37]. After a median follow-up of 45 months, median overall and event-free survivals were longer in the 80 patients with than in the 82 patients without HLA-identical siblings ( 80 months versus 54 months, $P=0.01$; and 35 months versus 29 months, $P=0.02$, respectively). Similar results have been reported in an EBMT-sponsored prospective trial using similar conditioning for autologous and allogeneic HSCT [38].

\section{Perspectives}

Further improvements in the outcome of RIC-HSCT require reinforcing GVT effects while limiting the deleterious effects of GVHD, and in particular acute GVHD.

Strategies aimed at increasing GVT effects are investigating combining disease-targeted therapies such as imatinib, thalidomide, bortezomib, rituximab, azacytidine, or radiolabeled monoclonal antibodies as part of the preparative regimen or as maintenance strategies.

One of the biggest challenges for the future of RIC allogeneic HSCT will be to develop protocols to better prevent acute GVHD. Potential approaches include various forms of T-cell depletion of the graft or in vivo T-cell depletion, as well as the use of immuno-regulatory cells such as mesenchymal stem cells, NK/T cells, or regulatory T cells.

Nonmyeloablative HSCT with CD8-depleted grafts has been evaluated by several groups, resulting in a lower incidence of severe acute GVHD [39]. However, in a recent phase II prospective randomized trial comparing patients receiving unmanipulated or CD8-depleted grafts after nonmyeloablative conditioning, Willems et al. reported a similar incidence of acute GVHD, albeit with less severe acute GVHD, but a higher risk of graft rejection in the CD8-depleted arm [40]. Based on similar concepts, other groups have proposed to reduce GVHD after RIC conditioning by performing in vivo T-cell depletion with ATG or alemtuzumab. These protocols have achieved their goal but have been associated with a higher risk of relapse [41].

Another approach aimed at reducing the incidence of acute GVHD has been developed by the Stanford group. Based on murine experiments, the authors investigated a novel nonmyeloablative regimen that favored the presence of a high proportion of regulatory NK-T cells [42]. This 
regimen consisted of TLI ( $8 \mathrm{~Gy}$ ) and ATG (Thymoglobulin, $7.5 \mathrm{mg} / \mathrm{kg}$ total dose), and postgrafting immunosuppression with MMF and CSP. The first results in 110 patients with various hematological malignancies indicated that this regimen was indeed associated with a low incidence of grade II-IV acute GVHD (<10\%), while GVT effects were apparently preserved.

Other strategies aimed at decreasing the incidence of acute GVHD have focused on mesenchymal stem cells (MSC). MSC are multipotent progenitors within the bone marrow capable of differentiating into various connective tissues and of modulating immune responses by inhibiting T-cell proliferation. Several groups have evaluated the potential of MSC infusions to prevent or to treat acute GVHD after allogeneic HSCT [43]. Recently, Baron et al. have investigated the impact on GVHD occurence of MSC co-infusion in 20 patients receiving HLA-mismatched allogeneic grafts after nonmyeloablative conditioning [44]. Compared with 16 similar historical controls, MSC co-transplantation was associated with a lower incidence of severe acute GVHD, lower NRM and improved survival.

Other potential avenues for limiting the risk of acute GVHD are based on regulatory $\mathrm{T}$ cells $\left(\mathrm{T}_{\text {reg }}\right)$. These immuno-regulatory cells are thought to promote allogeneic tolerance. Rezvani et al. have suggested that the graft $T_{\text {reg }}$ content and the rate of $\mathrm{T}_{\text {reg }}$ reconstitution after transplantation may predict the risk of GVHD after HSCT with myeloablative conditioning [45]. Therefore, manipulating $\mathrm{T}_{\text {reg }}$ numbers early after transplantation may provide a new approach to control acute GVHD in myeloablative as well as RIC settings. $\mathrm{T}_{\text {reg }}$ infusions after transplantation as well as $\mathrm{T}_{\text {reg }}$ number expansion by immunoregulatory drugs such as rapamycine are under investigation.

\section{Conclusion}

Reduced intensity conditioning (RIC) regimens have allowed performing allogeneic hematopoietic stem cell transplantation (HSCT) in patients for whom conventional myeloablative allogeneic HSCT would have been associated with unacceptable risks of non-relapse mortality. This approach relies mainly on graft-versus-tumor effects for tumor eradication. Results after RIC HSCT are generally encouraging in patient with acute myeloid leukemia in complete remission, with myelodysplastic syndromes not in transformation, with indolent and chemotherapy-sensitive aggressive lymphoma and with chronic lymphoid leukemia. At the opposite, advanced aggressive diseases such as acute leukemia not in complete remission, chemotherapy-refractory high-grade lymphoma or multiple myeloma are less responsive to this therapeutic approach alone. For such diseases, previous cytoreduction by chemotherapy or autologous HSCT is generally required. Occurrence of chronic GVHD seems to correlate with a lower risk of relapse and better progression-free survival. However, acute GVHD is not associated with GVT effects after nonmyeloablative conditioning and remains a serious complication of RIC HSCT. Current research attempts at developing new methods to control it.

\section{Disclosure}

The authors have no potential conflict of interest.

\section{Acknowledgments}

SS is Research Fellow (Televie grant) and FB Senior Research Associate of the National Fund for Scientific Research (F.R.S.-FNRS) Belgium.

\section{References}

[1] Little MT, Storb R. History of haematopoietic stem-cell transplantation. Nat. Rev. Cancer 2002;2:231-8.

[2] Weiden PL, Sullivan KM, Flournoy N, Storb R, Thomas ED. Antileukemic effect of chronic graft-versus-host disease: contribution to improved survival after allogeneic marrow transplantation. N. Engl. J. Med. 1981;304:1529-33.

[3] Horowitz MM, Gale RP, Sondel PM, et al. Graft-versus-leukemia reactions after bone-marrow transplantation. Blood 1990;75: 555-62.

[4] Kolb HJ. Graft-versus-leukemia effects of transplantation and donor lymphocytes. Blood 2008;112:4371-83.

[5] Baron F, Petersdorf EW, Gooley T, et al. What is the role for donor natural killer cells after nonmyeloablative conditioning? Biol. Blood Marrow Transplant. 2009;15:580-8.

[6] Miklos DB, Kim HT, Miller KH, et al. Antibody responses to $\mathrm{H}-\mathrm{Y}$ minor histocompatibility antigens correlate with chronic graftversus-host disease and disease remission. Blood 2005;105:2973-8.

[7] Nishida T, Hudecek M, Kostic A, et al. Development of tumor-reactive $\mathrm{T}$ cells after nonmyeloablative allogeneic hematopoietic stem cell transplant for chronic lymphocytic leukemia. Clin. Cancer Res. 2009;15:4759-68.

[8] Ruggeri L, Capanni M, Urbani E, et al. Effectiveness of donor natural killer cell alloreactivity in mismatched hematopoietic transplants. Science 2002;295:2097-100.

[9] Verheyden S, Schots R, Duquet W, Demanet C. A defined donor activating natural killer cell receptor genotype protects against leukemic relapse after related HLA-identical hematopoietic stem cell transplantation. Leukemia 2005;19:1446-51.

[10] Kahl C, Storer BE, Sandmaier BM, et al. Relapse risk in patients with malignant diseases given allogeneic hematopoietic cell transplantation after nonmyeloablative conditioning. Blood 2007;110: 2744-8.

[11] Giralt S, Estey E, Albitar M, et al. Engraftment of allogeneic hematopoietic progenitor cells with purine analog-containing chemotherapy: harnessing graft-versus-leukemia without myeloablative therapy. Blood 1997;89:4531-6.

[12] Baron F, Sandmaier BM. Nonmyeloablative Transplantation. In: Soiffer RJ, editor. Hematopoietic Stem Cell Transplantation. Totowa: Humana Press; 2008. p. 349-74.

[13] Baron F, Baker JE, Storb R, et al. Kinetics of engraftment in patients with hematologic malignancies given allogeneic hematopoietic cell transplantation after nonmyeloablative conditioning. Blood 2004;104:2254-62.

[14] Blaise D, Tabrizi R, Le Corroller AG, et al. Prospectives randomized comparison of reduced intensity (FLU-BU-ATG), non-myeloablative (FLU-TBI) conditioning for genoidentical allo-SCT: a clinical, economical multicenter ITAC study [abstract]. ASH Annual Meeting Abstracts 2009;114:193.

[15] Mohty M, Labopin M, Janssen JJWM, et al. Comparison of low dose total body irradiation (TBI)-based reduced intensity conditioning (RIC) vs. chemotherapy-based RIC prior to allogeneic stem cell transplantation (allo-SCT) from an HLA identical sibling donor for Acute Myeloid Leukemia (AML) in first complete remission (CR1): a retrospective analysis of 1200 Patients from the Acute Leukemia Working Party of EBMT [abstract]. ASH Annual Meeting Abstracts 2009; $114: 1190$.

[16] Baron F, Maris MB, Sandmaier BM, et al. Graft-versus-tumor effects after allogeneic hematopoietic cell transplantation with nonmyeloablative conditioning. J. Clin. Oncol. 2005;23:1993-2003.

[17] McSweeney PA, Niederwieser D, Shizuru JA, et al. Hematopoietic cell transplantation in older patients with hematologic malignancies: replacing high-dose cytotoxic therapy with graft-versus-tumor effects. Blood 2001;97:3390-400. 
[18] Mielcarek M, Martin PJ, Leisenring W, et al. Graft-versus-host disease after nonmyeloablative versus conventional hematopoietic stem cell transplantation. Blood 2003;102:756-62.

[19] Valcarcel D, Martino R, Caballero D, et al. Sustained remissions of high-risk acute myeloid leukemia and myelodysplastic syndrome after reduced-intensity conditioning allogeneic hematopoietic transplantation: chronic graft-versus-host disease is the strongest factor improving survival. J. Clin. Oncol. 2008;26:577-84.

[20] Sandmaier BM, Mackinnon S, Childs RW. Reduced intensity conditioning for allogeneic hematopoietic cell transplantation: current perspectives. Biol. Blood Marrow Transplant. 2007;13: 87-97.

[21] Larosa F, Marmier C, Robinet E, et al. Peripheral T-cell expansion and low infection rate after reduced-intensity conditioning and allogeneic blood stem cell transplantation. Bone Marrow Transplant. 2005;35:859-68.

[22] Junghanss C, Marr KA, Carter RA, et al. Incidence and outcome of bacterial and fungal infections following nonmyeloablative compared with myeloablative allogeneic hematopoietic stem cell transplantation: a matched control study. Biol. Blood Marrow Transplant. 2002;8:512-20.

[23] Aoudjhane M, Labopin M, Gorin NC, et al. Comparative outcome of reduced intensity and myeloablative conditioning regimen in HLA identical sibling allogeneic haematopoietic stem cell transplantation for patients older than 50 years of age with acute myeloblastic leukaemia: a retrospective survey from the Acute Leukemia Working Party (ALWP) of the European group for Blood and Marrow Transplantation (EBMT). Leukemia 2005;19:2304-12.

[24] Dreger P, Brand R, Milligan D, et al. Reduced-intensity conditioning lowers treatment-related mortality of allogeneic stem cell transplantation for chronic lymphocytic leukemia: a populationmatched analysis. Leukemia 2005;19:1029-33.

[25] Martino R, Iacobelli S, Brand R, et al. Retrospective comparison of reduced-intensity conditioning and conventional high-dose conditioning for allogeneic hematopoietic stem cell transplantation using HLA-identical sibling donors in myelodysplastic syndromes. Blood 2006;108:836-46.

[26] Gyurkocza B, Storb R, Storer BE, et al. Nonmyeloablative allogeneic hematopoietic cell transplantation in patients with acute myeloid leukemia. J. Clin. Oncol. 2010;28:2859-67.

[27] Marks DI, Wang T, et al. Comparison of outcomes for NonMyeloablative (NMA) and Myeloablative (MA) conditioning for adults with Acute Lymphoblastic Leukaemia (ALL) in first and second Complete Remission (CR): a Center for International Blood and Marrow Transplant Research (CIBMTR) Analyisis. ASH Annual Meeting Abstracts 2009;114:872.

[28] Laport GG, Sandmaier BM, Storer BE, et al. Reduced-intensity conditioning followed by allogeneic hematopoietic cell transplantation for adult patients with myelodysplastic syndrome and myeloproliferative disorders. Biol. Blood Marrow Transplant. 2008;14:246-55.

[29] Kroger N, Holler E, Kobbe G, et al. Allogeneic stem cell transplantation after reduced-intensity conditioning in patients with myelofibrosis: a prospective, multicenter study of the Chronic Leukemia Working Party of the European Group for Blood and Marrow Transplantation. Blood 2009;114:5264-70.

[30] Baron F, Storb R, Storer BE, et al. Factors associated with outcomes in allogeneic hematopoietic cell transplantation with nonmyeloablative conditioning after failed myeloablative hematopoietic cell transplantation. J. Clin. Oncol. 2006;24:4150-7.

[31] Escalon MP, Champlin RE, Saliba RM, et al. Nonmyeloablative allogeneic hematopoietic transplantation: a promising salvage therapy for patients with non-Hodgkin's lymphoma whose disease has failed a prior autologous transplantation. J. Clin. Oncol. 2004;22: 2419-23.

[32] Maris MB, Sandmaier BM, Storer BE, et al. Allogeneic hematopoietic cell transplantation after fludarabine and $2 \mathrm{~Gy}$ total body irradiation for relapsed and refractory mantle cell lymphoma. Blood 2004;104:3535-42.

[33] Morris E, Thomson K, Craddock C, et al. Outcomes after alemtuzumab-containing reduced-intensity allogeneic transplantation regimen for relapsed and refractory non-Hodgkin lymphoma. Blood 2004;104:3865-71.

[34] Robinson SP, Sureda A, Canals C, et al. Reduced intensity conditioning allogeneic stem cell transplantation for Hodgkin's lymphoma: identification of prognostic factors predicting outcome. Haematologica 2009;94:230-8.

[35] Sorror ML, Storer BE, Maloney DG, et al. Outcomes after allogeneic hematopoietic cell transplantation with nonmyeloablative or myeloablative conditioning regimens for treatment of lymphoma and chronic lymphocytic leukemia. Blood 2008;111:446-52.

[36] Garban F, Attal M, Michallet M, et al. Prospective comparison of autologous stem cell transplantation followed by dose-reduced allograft (IFM99-03 trial) with tandem autologous stem cell transplantation (IFM99-04 trial) in high-risk de novo multiple myeloma. Blood 2006;107:3474-80.

[37] Bruno B, Rotta M, Patriarca F, et al. A comparison of allografting with autografting for newly diagnosed myeloma. N. Engl. J. Med. 2007;356:1110-20.

[38] Gahrton G, Bjorkstrand B, Iacobelli S, et al. Tandem autologous (ASCT)/Allogeneic Reduced Intensity Conditioning Transplantation (RIC) with identical sibling donor versus ASCT in previously untreated Multiple Myeloma (MM): long term follow up of a prospective controlled trial by the EBMT [abstract]. ASH Annual Meeting Abstracts 2009;114:52.

[39] Baron F, Frere P, Baudoux E, et al. Low incidence of acute graftversus-host disease after non-myeloablative stem cell transplantation with CD8-depleted peripheral blood stem cells: an update. Haematologica 2003;88:835-7.

[40] Willems E, Baron F, Baudoux E, et al. Non-myeloablative transplantation with CD8-depleted or unmanipulated peripheral blood stem cells: a phase II randomized trial. Leukemia 2009;23: 608-10.

[41] Crawley C, Lalancette M, Szydlo R, et al. Outcomes for reducedintensity allogeneic transplantation for multiple myeloma: an analysis of prognostic factors from the Chronic Leukaemia Working Party of the EBMT. Blood 2005;105:4532-9.

[42] Kohrt HE, Turnbull BB, Heydari K, et al. TLI and ATG conditioning with low risk of graft-versus-host disease retains antitumor reactions after allogeneic hematopoietic cell transplantation from related and unrelated donors. Blood 2009;114:1099-109.

[43] Le Blanc K, Frassoni F, Ball L, et al. Mesenchymal stem cells for treatment of steroid-resistant, severe, acute graft-versus-host disease: a phase II study. Lancet 2008;371:1579-86.

[44] Baron F, Lechanteur C, Willems E, et al. Cotransplantation of mesenchymal stem cells might prevent death from graft-versushost disease (GVHD) without abrogating graft-versus-tumor effects after HLA-mismatched allogeneic transplantation following nonmyeloablative conditioning. Biol. Blood Marrow Transplant. 2010;16:838-47.

[45] Rezvani K, Mielke S, Ahmadzadeh M, et al. High donor FOXP3positive regulatory T-cell (Treg) content is associated with a low risk of GVHD following HLA-matched allogeneic SCT. Blood 2006;108: 1291-7. 